\title{
DESIGN AND SIMULATION OF MICROFLUIDIC PASSIVE MIXER WITH GEOMETRIC VARIATION
}

\author{
Shubha Jain ${ }^{1}$, H. N. Unni ${ }^{2}$ \\ ${ }^{1}$ Research Scholar, Department of Biomedical Engineering, Indian Institute of Technology Hyderabad, India \\ ${ }^{2}$ Assistant Professor, Department of Biomedical Engineering, Indian Institute of Technology Hyderabad, India \\ ${ }^{1}$ bm14resch11005@iith.ac.in, ${ }^{2}$ harikrishnan@iith.ac.in
}

\begin{abstract}
Microfluidic designs are advantageous and are extensively used in number of fields related to biomedical and biochemical engineering. The objective of this paper is to perform numerical simulations to optimize the design of microfluidic mixers in order to achieve optimum mixing. In the present study, fluid mixing in different type of micro channels has been investigated. Numerical simulations are performed in order to understand the effect of channel geometry parameters on mixing performance. A two dimensional "T shaped" passive microfluidic mixer is restructured by employing the rectangular shaped obstacles in the channel to improve the mixing performance. The impact of proper placement of obstacles in the channel is demonstrated by applying the leakage concept. It has been observed that, the channel design with non-leaky obstacles (i.e. without leaky barriers) has presented better mixing performance in contrast to channel design with leaky obstacles (i.e. leaky barriers) and channel design without obstacles. The mixing occurs by virtue of secondary flow and generation of vortices due to curling of fluids in the channel on account of the presence of obstacles. This passive mixer has achieved complete mixing of fluids in few seconds or some milliseconds, which is certainly acceptable to utilize in biological applications such as cell dynamics, drug screening, toxicological screening and others.
\end{abstract}

Keywords: Microfluidic Mixing, Passive Mixer, Microchannel, Numerical Simulation

\section{INTRODUCTION}

Microfluidics is an interdisciplinary field that interconnects physics, chemistry, engineering and biotechnology. Channels with dimensions of tens to several hundred micrometers are employed to manipulate the small amount of fluid. Fluid mixing is one of the seminal applications of chip scale micro devices. Generally, microfluidics mixers are classified as "active mixers" and "passive mixers" [1], [2]. Active mixers demand external force fields, such as thermal [3], acoustic [4], magnetic [5] and electro kinetic [6] to strengthen mixing efficiency. Conversely, passive mixers employ no external energy input and judicial selection of device geometry play dominant role for getting effective mixing. These designs account for several advantages such as cost effectiveness, reduced complexity, ease of fabrication etc. Additionally, active mixing designs when used with improper selection of operating parameters could be harmful for biological samples. Hence passive mixers offer some flexibility in this regard.

Flow physics changes dramatically at micro-scale, and it is really crucial to understand the effects of dominating factors such as laminar flow, diffusion, surface tension, fluidic resistance and surface area to volume ratio [7], [8] in order to work on microfluidic designs. The main aim of microfluidic mixing devices is to acquire complete and optimum mixing of two or more samples in micro-scale devices. However, achieving effective mixing of more than one fluid is very challenging task, owing to the limitation induced by reduced convection in micro-channels.
Molecular diffusion plays a dominant role in passive microfluidic mixers [9] unlike the case of convectiondominated flows and therefore viscous force has immense effect as low Reynolds number is considered [10], [11]. Many researchers have proposed numerous designs that focused on effective passive mixing [12]-[19]. Lee et al. [12] has investigated the mixing efficiency by using the different type of geometric barriers which shows acceptable mixing. Wang et al. [14] presented a design for mixing of fluids streams in "Y" type micro channels by using cylindrical shaped obstacles for enhancing mixing performance. The significant effect of proper placement and position of obstacles were presented. Wong et.al [17] designed and fabricated micro-mixers as well as investigated their feasibility as a rapid mixing micromixer. Das et al. [18] presented a computational study of fluid mixing in three different type of micro-channels. They have shown that impact of geometry parameters and placement of obstacle by creating swirling flow in the channel for improving mixing performance. Liu et al. [19] reported a three-dimensional structure of serpentine design with $\mathrm{C}$ shaped repeating unit to improve mixing.

In this present work, we have designed a ' $\mathrm{T}$ ' shaped passive microfluidic mixer to investigate microfluidic mixing of fluids. We have examined that the mixing performance is refined with appropriate choice of placement of obstacles. A comparison of mixing performance has been made among three mixing designs (i) without barriers, (ii) with leaky barriers and (iii) without leaky barriers as shown in fig (1). 
Consequently, complete mixing is achieved at the end of channel reservoir in case of barrier without leakage. All simulations presented in this work have been performed by COMSOL Multiphysics software. The results from the present study could be useful in the design of microfluidic mixers applied for drug screening and toxicological screening.

\section{MODELING}

\subsection{Microchannel Geometry}

Two dimensional straight ' $\mathrm{T}$ ' shaped micro-channel were designed as shown in fig [1]. They were reformed by embedding obstacles in the channel. These obstacles are positioned in two different ways, named as leakage barriers and without leakage barriers as demonstrated in fig [1 (b, c)]. The total axial length of channel is $190 \mu \mathrm{m}$, width of inlet is $10 \mu \mathrm{m}$, width of mixing channel is $40 \mu \mathrm{m}$ and number of barriers are 10 which are placed in $25 \mu \mathrm{m}$ displacement.

Rectangular barriers are placed with angular displacement of $\pm 45^{\circ}$. The sub conjugated image barriers have been placed with $10 \mu \mathrm{m}$ gap and anti-image barriers have been placed with $15 \mu \mathrm{m}$ gap. The upper and lower rows contain same number of barriers. The barriers were distributed uniformly along the direction of the flow through the channels.

(a)

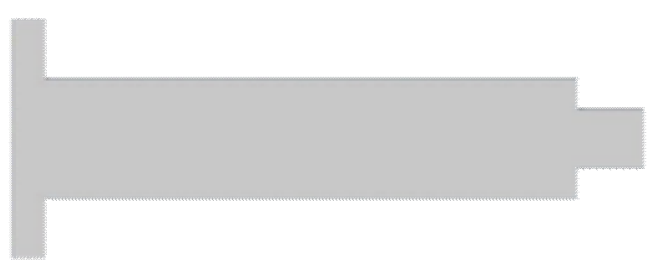

(b)

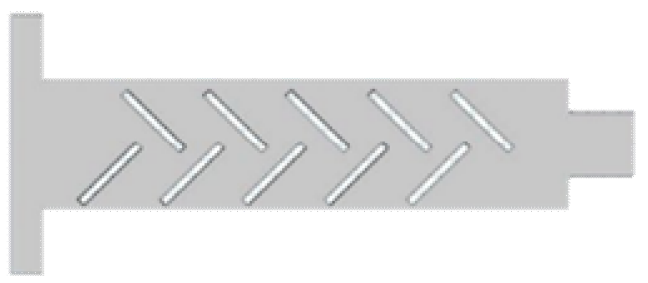

(c)

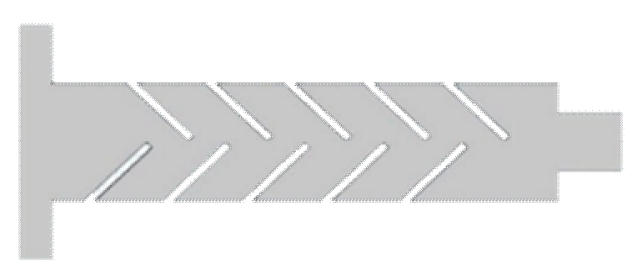

Fig -1: Geometry of channel (a) without barrier (b) with leaky barrier (c) without leaky barrier

Fluids with different concentration enter through upper and lower inlets and met at ' $\mathrm{T}$ ' junction. Afterwards, fluid mixing proceeds in the channel, where the concentration from one inlet (upper) is designated as " 1 " ( $\left.\mathrm{mol} / \mathrm{m}^{3}\right)$ concentration and another from lower inlet is designated as " 0 " $\left(\mathrm{mol} / \mathrm{m}^{3}\right)$. The average concentration of fluids in perfect mixed state would be $0.5\left(\mathrm{~mol} / \mathrm{m}^{3}\right)$.

\subsection{Flow and Concentration Field Modeling}

In particular, modeling is done by considering fluid as an incompressible Newtonian liquid in microchannel. Fluid flow and concentration fields can generally be described by the Navier-Stokes equation [20] and Convection Diffusion equation [17] respectively. Mathematical expression of Navier-Stokes and Continuity equations are as follows:

$$
\begin{aligned}
& \rho \frac{\partial V}{\partial t}+\rho(V \cdot \nabla) V=-\nabla p+\mu \nabla^{2} V+F \\
& \nabla \cdot V=0
\end{aligned}
$$

where $V$ is the fluid velocity vector, $\rho$ is the fluid density, $p$ is the pressure, $\mu$ is the dynamic viscosity of the fluid, $F$ represents body forces per unit volume. On the other hand, the Convection Diffusion equation is mathematically expressed as:

$$
\frac{\partial c}{\partial t}+(V . \nabla) c=D \nabla^{2} c
$$

where $c$ is the concentration constant and $D$ is the diffusion constant of the species.

Three regimes of flow exit according to the elementary theory of fluid dynamics, which are represented by Reynolds number ( $\mathrm{Re})$, is a dimensionless parameter. It is expressed as:

$$
\operatorname{Re}=\frac{\rho u L_{c}}{\mu}
$$

where $u$ is the fluid flow velocity and $L_{c}$ is the characteristic length of the flow. The flow is considered to be laminar flow when Reynolds number is less than 2300. It would be turbulent flow when Reynolds number is greater than 4000 . Between the laminar and turbulent flow (2300-4000), the flow is considered in a transitional regime. In case of microfluidic flows, Reynolds number falls down to regime of $<1$ in many cases. These kind of flows are normally referred to as creeping flows. The optimization is carried out by composing 14802, 10690 number of mesh elements in the designs with leaky barriers and without leaky barriers respectively.

\section{RESULTS AND DISCUSSION}

Primarily, the $\mathrm{T}$ shape channel without barriers has been simulated. Form the simulation result it is clarified that fluids are not getting mixed properly in the channel as shown in fig [2(a)], as there is a strong concentration gradient that exists at channel exit. In order to overcome this, the channel is reformed and simulated by constructing the rectangular type obstacles. These obstacles are placed by using the concept of leakage and without leakage barriers as presented in fig $[2(b, c)]$. These modified designs are simulated at different velocity at constant diffusion coefficient $\left(1 \times 10^{-10} \mathrm{~m}^{2} / \mathrm{s}\right)$ conditions. It is inferred that length of mixing increases with increase in inlet velocity and at the same time mixing time reduces, however it happens within a particular range of velocity and in addition, it varies with the geometry. 
The simulation results show that the mixing performance is improved by employing obstacles in the channel as reported in the $[14,15]$. Channel designs without leaking barriers are shown to provide better mixing compared to designs with leaky barriers. However, if barriers are leaky in nature, this will drastically affect the mixing and consequently fluids will not get mixed properly as presented in fig [2(b)]. By contrast, in case of the channel without leaky barrier, we have achieved complete mixing as shown in fig [2(c)]. The comparison between both types of designs is demonstrated in table [1] in the form of simulation results. The table shows the clear demarcation in the performance of the two designs. Thus, the impact of barriers and appropriate placement of barriers both can bear significant role in mixing channel.

(a)

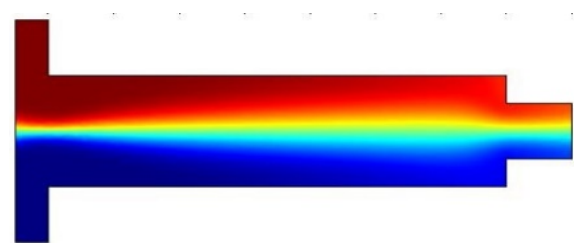

(b)

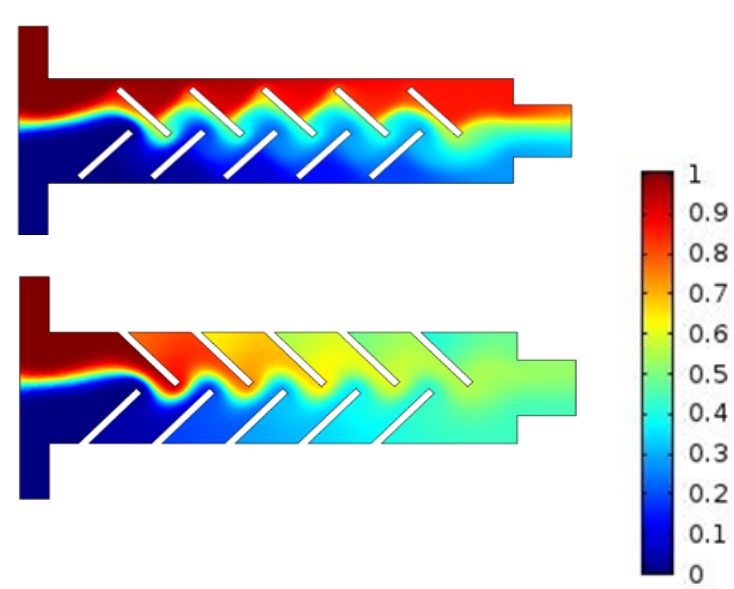

Fig-2: Simulation results of T-type mixers where the inlet velocity is $40 \times 10^{-5} \mathrm{~m} / \mathrm{s}$ (a) without barrier (b) with leaky barrier (c) without leaky barrier

Due to the placement of obstacles, cross sectional area of channel reduces on account of which flow velocity gets amplified. In the present study, barriers are placed with an angular displacement of $45^{\circ}$. After injecting the fluids, the flow gets curled on account of barriers and create secondary flows which help to produce vortices in the channel, thus mixing is significantly enhanced. The design without leaky barriers achieved complete mixing in around $1.9 \mathrm{~s}$, and this demonstrate the sufficient potential for evolving acceptable mixing in microfluidic channels as shown in fig [2(c)].
Table -1: Concentration in channel with leaky barrier (a-c). Concentration in channel without leaky barrier (i-iii).

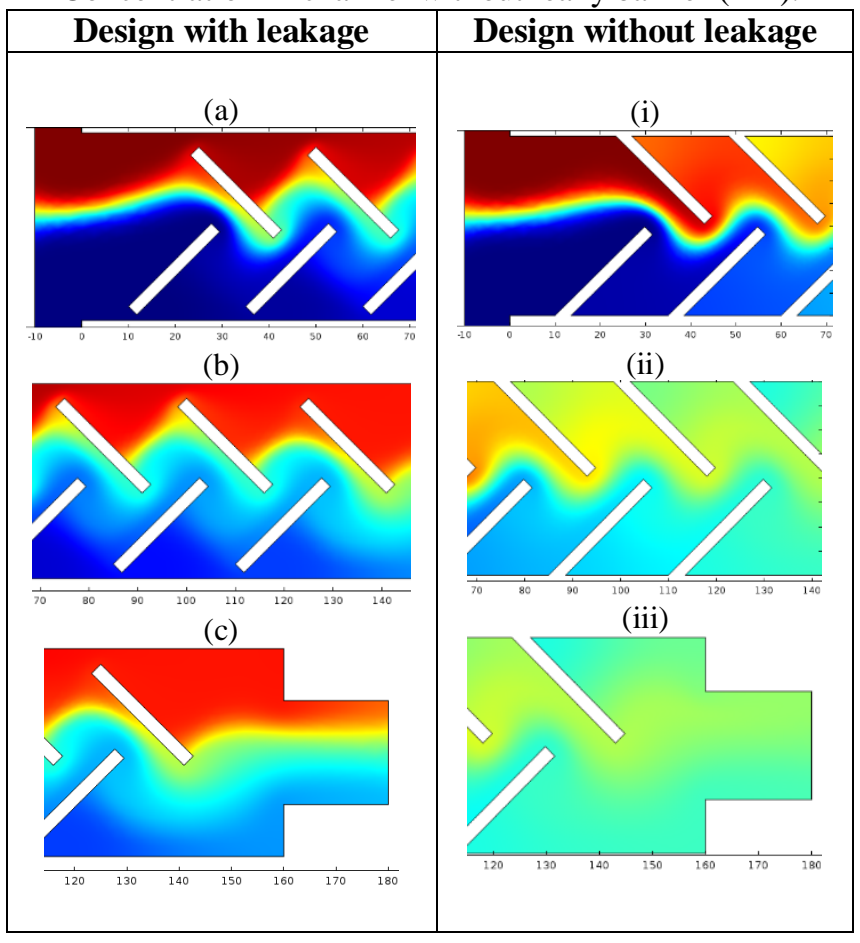

Comparison between channel designs is represented by plotting the graph of concentration difference $\left(\varepsilon=c_{\max } c_{\min }\right)$ versus positions along channel length (at different positions from inlet) as shown in fig [3]. These points have been taken at the mixing time of channel without leaky barrier i.e. 1.9s. This is done mainly to demarcate the difference between the two designs evaluated in the present study. It is evident that near perfect mixing is achieved at a distance of $180 \mu \mathrm{m}$ from inlet, where the performance of leaky design is poor. The performance of the non-leaky design is further evaluated in fig [4], which depicts the concentration profile at channel exit (corresponding to different times). It can be note that concentration profile nearly flattens out to a value of 0.5 at $1.9 \mathrm{sec}$.

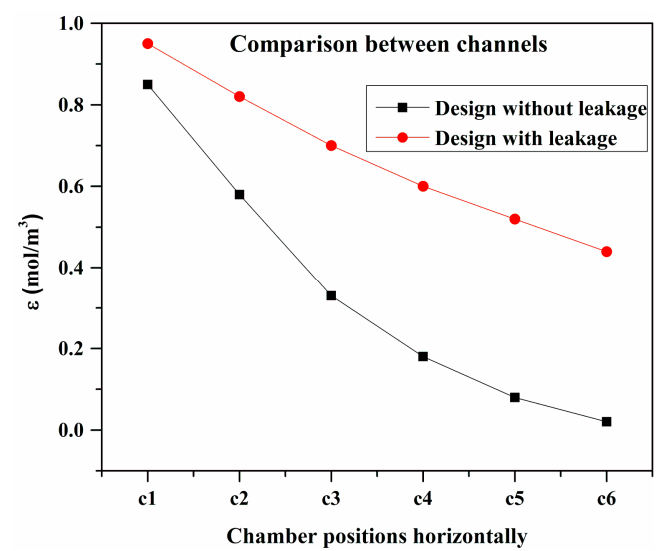

Fig -3: Comparison between leaky and non-leaky channel designs: Concentration difference $\left(\varepsilon=c_{\max } c_{\min }\right)$ plotted

against various positions along the channel length represented as c1, c2, c3, c4, c5, c6 at a distance of $30 \mu \mathrm{m}$, $60 \mu \mathrm{m}, 90 \mu \mathrm{m}, 120 \mu \mathrm{m}, 150 \mu \mathrm{m}, 180 \mu \mathrm{m}$ from entrance (after inlet) respectively. 


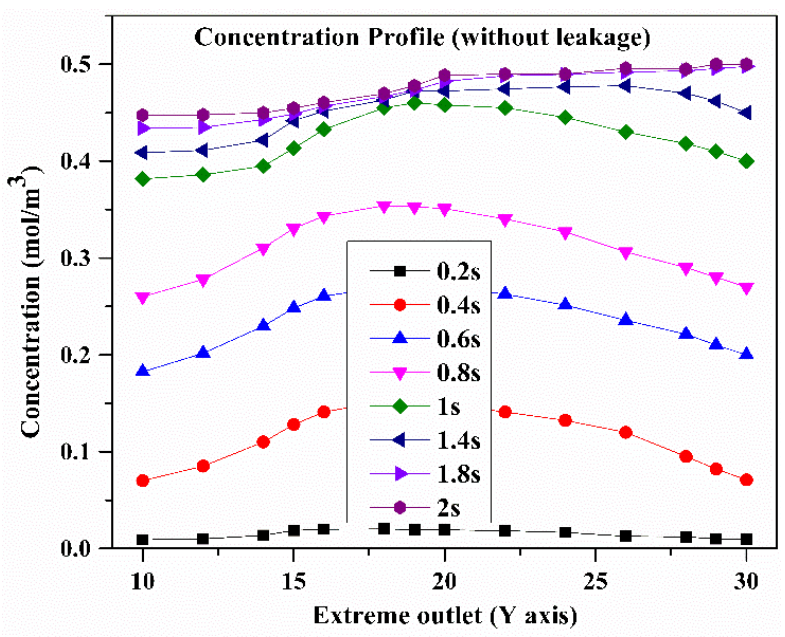

Fig -4: Concentration profile at channel exit corresponding to design with non-leaky barrier at outlet at different time

\section{CONCLUSIONS}

Numerical simulation is performed to investigate the mixing performance of passive mixers. The significant impact of barriers and the proper placement of barriers is recognized by the simulation. The channel with leaky barrier is incapable to achieve complete mixing due to leakage of fluid from upper and lower part of channel. In a nutshell, complete mixing is achieved in a short duration by the virtue of flow rotation and secondary flows by cause of proper placement of rectangular barrier in channel. In addition, this channel could be useful in the development of microfluidic devices targeted to cell-drug mixing analysis.

\section{ACKNOWLEDGEMENT}

The authors are thankful to IITH and MHRD for their immense support.

\section{REFERENCES}

[1] H, H. Lowe, and F. Schonfeld, "Micromixers-a review on passive and active mixing principles" Chem. Eng. Sci., vol. 60, pp. 2479-2501, 2005.

[2] N. T. Nguyen and Z. Wu, "Micromixer: a review", J. Micromech. Microeng. vol.15, pp. R1-R16, 2005.

[3] J. Tsai, L. Lin, "Active microfluidic mixer and gas bubble filter driven by thermal bubble micropump", Sens. Actuators A, vol. 97/98, pp. 665-671, 2002.

[4] Z. Yang, H. Goto, M. Matsumo, R. Maeda, "Ultrasonic micromixer for microfluidic systems", Sens. Actuators A, vol. 93, pp, 266-272, 2001.

[5] H. Bau, J. Zhong, M. Yi, "A minute magneto hydro dynamic (MHD) mixer", Sens. Actuators B, vol. 79, pp. 207-21, 2001.

[6] S. Jacobson, T. McKnight, J. Ramsey, "Microfluidic devices for electrokinematically driven parallel and serial mixing", Anal. Chem., vol. 71, pp. 4455-4459, 1999.

[7] Gravesen P, Branebjerg J, Jensen O., "Micro-fluidicsa review", J. Micromech. Microeng, vol. 3, pp. 168-82, 1993.
[8] Purcell E., "Life at low Reynolds number", American Journal of Physics, Vol. 45, pp. 3-11, 1977.

[9] Wang H., Iovenitti P., Harvey E., Masood S., "Numerical investigation of mixing in micro-channels with patterned grooves", J. Micromech. Microgen, vol. 13, pp. 801-808, 2003.

[10] W. Ehrfeld, V. Hessel and H. Lowe, Microreactors, "New Technology for Modern Chemistry", New York: Wiley-Vch, 2000.

[11] K. Benz, K.-P. Jackel, K.-J. Regenauer, J. Schiewe, K. Drese, W. Ehrfeld, V. Hessel and H. Lowe, "Utilization of Micromixers for Extraction Processes", Chem. Eng. Technol., vol. 24, pp. 11, 2001.

[12]C.Y. Lee, C.F. Lin, M.F. Hung, R.H. Ma, C.H. Tsai, C.H. Lin and L.M. Fu, "Experimental and numerical investigation into mixing efficiency of micromixers with different geometric barriers, Materials Science Forum Vols. 505-507, pp 391-396, 2006.

[13] C. C. Hong, J. W. Choi and C. H. Ahn, "A novel inplane passive micromixer using Coanda effect Micro Total Analysis Systems, pp. 31-33, 2001.

[14]C. T. Wang and Y. C. Hu, "Mixing of liquids using obstacles in Y-type microchannels", Tamkang Journal of Science and Engineering, Vol. 13, No. 4, pp. 385394, 2010.

[15]H. Wang, P. Iovenitti, E. Harvey, and S. Masood, "Optimizing layout of obstacles for enhanced mixing in microchannels," Smart Materials and Structures, vol. 11, pp. 662-667, 2002.

[16] A. D. Stroock, S. K. W. Dertinger, A. Ajdari, I. Mezic, H. A. Stone and G. M. Whitesides, "Chaotic mixer for microchannels”, Science, vol. 295, pp. 647-651, 2002.

[17] Seck Hoe Wong, Michael C.L. Ward, Christopher W. Wharton, "Micro T-mixer as a rapid mixing micromixer", Sensors and Actuators B, vol. 100, pp. 359-379, 2004.

[18] Sankha Shuvra Das, Binay Kumar Patawari, P.K. Patowari and S Halder, "Computational analysis for mixing of fluids flowing through microchannels of different geometries" AIMTDR 2014, IIT Guwahati, Assam, India.

[19]R. H. Liu, M. A. Stremler, K. V. Sharp, M. G. Olsen, J. G. Santiago, R. J. Adrian, H. Aref and D. J. Beebe, "Passive mixing in a three dimensional Serpentine microchannel", J. MEMS, vol.9, pp. 190-197, 2000.

[20] J. Judy, D. Maynes, B.W. Webb, "Characterization of frictional pressure drop for liquid flows through microchannels", Int. J. Heat Mass Transfer, vol. 45, pp. 3477-3489, 2002.

\section{BIOGRAPHIES}

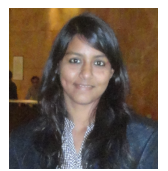

Ms. Shubha Jain is pursuing $\mathrm{PhD}$ from IIT HYDERABAD in the Department of Biomedical engineering.

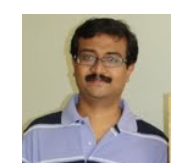

Dr. Harikrishnan Narayanan Unni is Assistant professor in IIT HYDERABAD the Department of Biomedical engineering. 